\title{
Cognitive function prior to systemic therapy and subsequent well-being in older breast cancer survivors: Longitudinal findings from the Thinking and Living with Cancer Study
}

\author{
Lindsay C. Kobayashi ${ }^{1}$ (ब) | Harvey Jay Cohen ${ }^{2}$ | Wanting Zhai ${ }^{3}$ | Xingtao Zhou ${ }^{3}$ \\ Brent J.Small ${ }^{4,5}$ ( ) | George Luta ${ }^{6}$ | Arti Hurria ${ }^{7}$ | Judith Carroll ${ }^{8}$ | \\ Danielle Tometich $^{9}$ (1) | Brenna C. McDonald ${ }^{10}$ | Deena Graham ${ }^{11}$ | \\ Heather S.L. Jim ${ }^{5}$ | Paul Jacobsen ${ }^{12}$ ～James C. Root ${ }^{13}$ (3) | Andrew J. Saykin ${ }^{10}$ | \\ Tim A. Ahles ${ }^{13}$ | Jeanne Mandelblatt ${ }^{3}$ \\ ${ }^{1}$ Department of Epidemiology, University of Michigan School of Public Health, Ann Arbor, Michigan \\ ${ }^{2}$ Center for the Study of Aging and Human Development, Duke University, Durham, North Carolina \\ ${ }^{3}$ Department of Oncology and Cancer Prevention and Control Program, Lombardi Comprehensive Cancer Center, Georgetown University, Washington, DC \\ ${ }^{4}$ School of Aging Studies, University of South Florida, Tampa, Florida \\ ${ }^{5}$ Moffitt Cancer Center, University of South Florida, Tampa, Florida \\ ${ }^{6}$ Department of Biostatistics, Bioinformatics, and Biomathematics and Cancer Prevention and Control Program, Lombardi Comprehensive Cancer Center, \\ Georgetown University, Washington, DC \\ ${ }^{7}$ Department of Medical Oncology and Therapeutics Research, City of Hope Comprehensive Cancer Center, Duarte, California \\ ${ }^{8}$ Department of Psychiatry and Biobehavioral Sciences, David Geffen School of Medicine, UCLA, Los Angeles, California \\ ${ }^{9}$ Department of Psychology, Indiana University-Purdue University Indianapolis, Indianapolis, Indiana \\ ${ }^{10}$ Center for Neuroimaging, Department of Radiology and Imaging Sciences and the Melvin and Bren Simon Cancer Center, Indiana University School of Medicine, \\ Indianapolis, Indiana \\ ${ }^{11}$ Hackensack University Medical Center, Hackensack, New Jersey \\ ${ }^{12}$ Healthcare Delivery Research Program, Division of Cancer Control and Population Sciences, National Cancer Institute, National Institutes of Health, Shady, \\ Maryland \\ ${ }^{13}$ Department of Psychiatry and Behavioral Sciences, Memorial Sloan-Kettering Cancer Center, New York, New York
}

\section{Correspondence}

Jeanne Mandelblatt, Lombardi Comprehensive Cancer Center, 3300 Whitehaven Blvd, Suite 4100, Washington, DC 20007, USA.

Email: mandelbj@georgetown.edu

Funding information

American Cancer Society, Grant/Award Number: IRG-92-152; National Cancer Institute, Grant/Award Numbers: F31CA220964, P30CA082709, P30CA51008, R01CA129769, R03CA241841, R35CA197289, T32CA117865; National Institute on Aging, Grant/Award Numbers: P30AG028716, P30AG10133, R01AG19771; U.S. National Library of Medicine, Grant/ Award Number: R01LM01136

\section{Abstract}

Objective: To investigate the relationships between self-reported and objectively measured cognitive function prior to systemic therapy and subsequent well-being outcomes over 24 months in older breast cancer survivors.

Methods: Data were from 397 women aged 60 to 98 diagnosed with non-metastatic breast cancer in the Thinking and Living with Cancer Study recruited from 2010-2016. Cognitive function was measured at baseline (following surgery, prior to systemic therapy) using neuropsychological assessments of attention, processing speed, and executive function (APE), learning and memory (LM), and the self-reported FACT-Cog scale. Well-being was measured using the FACT-G functional, physical, social, and emotional well-being domain scales at baseline and 12 and 24 months later, scaled from 0 (low) to 100 (high). Linear mixed-effects models assessed the 
relationships between each of baseline $A P E, L M$, and FACT-Cog quartiles with well-being scores over 24 months, adjusted for confounding variables.

Results: At baseline, older survivors in the lowest APE, LM, and FACT-Cog score quartiles experienced poorer global well-being than those in the highest quartiles. At 24 months, older survivors tended to improve in well-being, and there were no differences according to baseline APE or LM scores. At 24 months, mean global well-being was 80.3 (95\% Cl: 76.2-84.3) among those in the lowest vs 86.6 (95\% Cl: 83.1-90.1) in the highest FACT-cog quartile, a clinically meaningful difference of 6.3 points $(95 \%$ Cl: 1.5-11.1).

Conclusions: Among older breast cancer survivors, self-reported, but not objective cognitive impairments, were associated with lower global well-being over the first 2 years of survivorship.

\section{KEYWORDS}

aging, breast cancer, cancer, cancer survivors, cognitive function, oncology, quality of life, selfreports, well-being

\section{1 | INTRODUCTION}

Breast cancer survivors are the largest group of female cancer survivors in the United States, and three-quarters of these women are $\geq 60$ years old ("older") at diagnosis. ${ }^{1-3}$ While survival rates are excellent, older cancer survivors live with the effects of cancer treatments, potential aging-related losses of physical and cognitive function, multi-morbidity, and changes to financial, social, and living situations. ${ }^{4-8}$ In particular, cancer-related cognitive impairment has gained attention as a central concern of older survivors. ${ }^{9-11}$ Cognitive problems at the time of diagnosis are important: although they may not be clinically obvious, they may affect women's abilities to manage their health after treatment, and may have substantial effects on daily function and well-being. ${ }^{11-15}$

However, the longitudinal well-being outcomes associated with selfreported cognitive changes or neuropsychological deficits at the time of diagnosis among older women with breast cancer have not yet been established. Prior reports have been based on younger women, used cross-sectional data, and/or did not include cognitive measures prior to systemic therapy. ${ }^{11,15-19}$ Few studies have compared the associations between objective and self-reported cognitive function with longitudinal well-being in older breast cancer survivors. Self-reports may reflect subtle changes in cognitive function that are not detected by formal neuropsychological assessments. Similar to self-reports of general health, they may also reflect many factors, including psychological health, in addition to true underlying cognitive function. ${ }^{20-24}$ Self-reported and objective cognitive assessments have been reported to have poor correlations within cancer survivor and general population samples, ${ }^{24}$ but their comparative associations with outcomes that are important to older cancer survivors have rarely been investigated.

To fill these evidence gaps, we used data from a national, prospective study of non-metastatic breast cancer survivors aged $\geq 60$ years at the time of diagnosis: The Thinking and Living with Cancer (TLC) Study. We investigated the relationships between objective and self-reported cognitive function prior to systemic therapy (baseline) and changes in global, functional, physical, social, and emotional domains of well-being at 12 and 24 months later. We hypothesized that older breast cancer survivors with lower cognitive function prior to systemic therapy would have persistently lower well-being over time than those with higher cognitive function, and that these associations would be strongest for self-reports of cognitive function. ${ }^{24}$ These results are intended to help identify older breast cancer survivors who may be at risk for poorer well-being over time and to inform the development and targeting of strategies to improve care and well-being for this growing older breast cancer survivor population.

\section{2 | METHODS}

\subsection{Study design}

This study was conducted at Georgetown University and affiliated practices in the Washington, DC area, Memorial Sloan Kettering Cancer Center in New York, Moffitt Cancer Center in Tampa, City of Hope Comprehensive Cancer Center in Los Angeles, Hackensack University Medical Center in New Jersey, Indiana University (IU) School of Medicine in Indianapolis, and the University of California at Los Angeles (UCLA). IU and UCLA joined the study for laboratory support and IU for participant recruitment in 2016; data in this report are from the five other sites. All Institutional Review Boards approved the protocol (NCT03451383). Details about the study are provided elsewhere. ${ }^{25}$ The present analysis uses data from all participants enrolled from 2010 through 2016 who completed follow-up at 12- and 24-months post-baseline. 


\subsection{Study participants}

Eligible study participants were English-speaking women aged 60 years and over with a new, primary non-metastatic breast cancer (AJCC v.6 stage 0-3). Exclusion criteria were having a history of stroke, head injury, a major Axis I psychiatric disorder, or neurodegenerative disorder. Women were ineligible if they had a history of other cancers with active treatment in the past 5 years or any systemic therapy. Women were screened for ability to complete the study based on hearing, vision, and baseline MMSE scores of 24+ and WRAT-4 reading level of third grade or higher. The consent rate among eligible women was $39.2 \%$ (range across sites: $17.2 \%-83.9 \%$; median 67.6\%). Among those who remained alive and eligible for inclusion, follow-up rates were $75.2 \%$ and $74.9 \%$ at 12 months and 24 months, respectively. TLC also recruited frequency-matched controls (based on 5-year age groups, education level, race, and site) using the same eligibility criteria and assessments. Consent rate among eligible controls was $96.2 \%$ (range across sites: $92.8 \%$ to $100 \%$; median $95.0 \%$ ), with $89.2 \%$ retention at 12 months and $81.8 \%$ at 24 months (Figure 1).

\section{$2.3 \mid$ Measures}

\subsection{1 | Well-being outcomes}

The well-being outcomes were measured using the FACT-G functional, physical, social, and emotional domain scales at baseline and 12 and 24 months later, scaled from 0 to 100 with higher scores representing better well-being. ${ }^{26-28}$ The domains were considered separately, and combined to create a global score. As per Webster et al, ${ }^{26}$ minimum clinically important differences in scores on the 0 to 100 scale were considered to be: $\geq 3.6$ points (global well-being); $\geq 8.3$ points (functional well-being), $\geq 8.3$ points (physical well-being), and $\geq 12.5$ points (emotional well-being). General population and cancer survivor norms on these scales for US adults are available from Brucker et al. ${ }^{27}$

\subsection{2 | Neuropsychological assessments}

A neuropsychological assessment was administered during the baseline (pre-systemic therapy) study interview to assess domain-specific cognitive functions. The assessment included six tests of attention, processing speed, and executive functioning (collectively, APE) and five tests of learning and memory (collectively, LM). We used tests of cognitive domains that are sensitive to aging-related changes and that have established validity and reliability in older populations. ${ }^{25}$ Using principal components analysis, we previously confirmed the domain structure of the APE and LM measures and their reliability over time within this sample. ${ }^{25}$ We $z$-standardized the composite APE and LM scores using the baseline age- and education-group matched healthy control means and standard deviations (SDs), to give a mean of 0 and SD of 1 . We categorized the scores into quartiles, in order to compare changes in well-being over time among older women across the baseline distribution of cognitive function.

\subsection{3 | Self-reported cognitive function}

Self-reported cognitive function was assessed using the FACT-Cog scale at baseline. ${ }^{28}$ The FACT-Cog has excellent test-retest reliability and has been validated among breast cancer survivors as reflecting cognitive functions assessed by neuropsychological measures and quantitative electroencephalography, independently of depressive symptoms. $^{29}$ The FACT-Cog items assess perceived cognitive functioning over the past 7 days according to four sub-scales: (a) Perceived Cognitive Impairments; (b) Perceived Cognitive Abilities; (c) Impact on Quality of Life; and (d) Comments from Others. The total scale has a Chronbach's alpha of 0.96. A previous validation study indicates a minimum clinically important difference of 6.9 points on the FACTCog scale among breast cancer survivors. ${ }^{30}$

The range of the FACT-Cog scale was 0 (low) to 148 (high); we categorized scores into quartiles to allow comparability with estimates for well-being outcomes for individuals at equivalent locations on the distributions of each of FACT-Cog, APE, and LM scores. The quartile

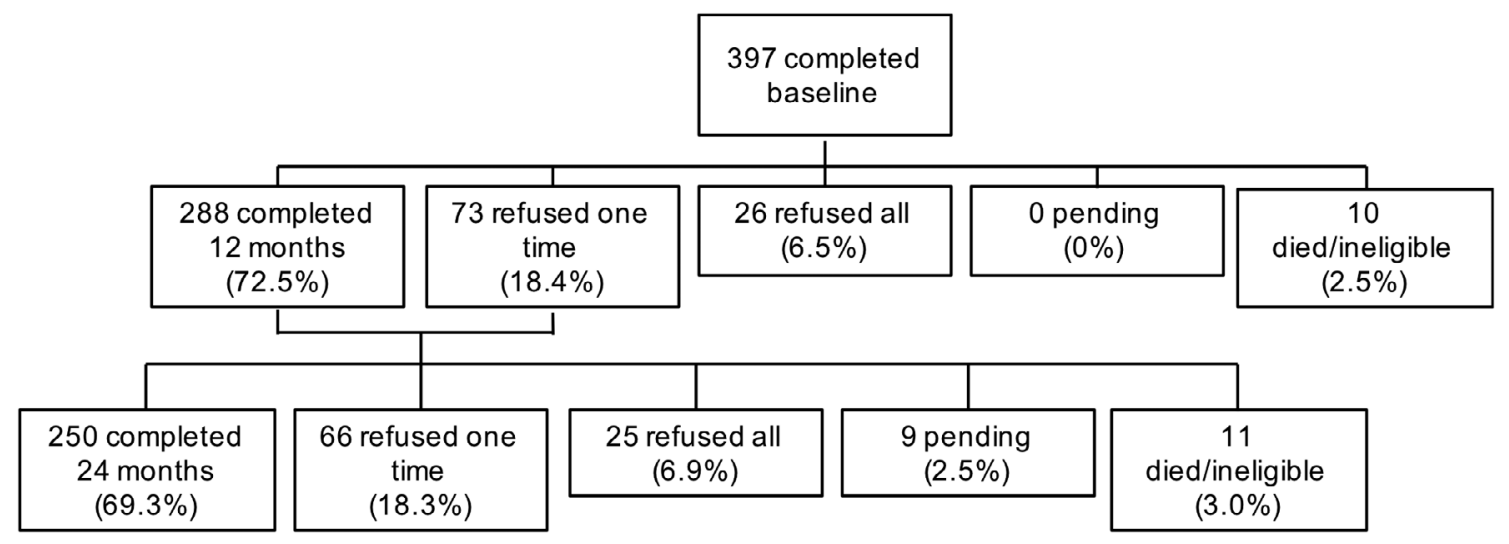

FIGURE 1 Study flow diagram, older breast cancer survivors, Thinking and Living with Cancer Study (recruitment to end of 2016 calendar year) 
cutoff values were <121 points (lowest), 121 to 134 points, 134 to 141 points, and $>141$ points (highest). Examination of the domainspecific scores within each quartile of the total FACT-Cog scale score indicated that the Perceived Cognitive Impairments and Perceived Cognitive Abilities domains were the primary drivers of variability in total scale score. The Impact on Quality of Life and Comments from Others domains demonstrated ceiling effects, with little differences in these domain-specific scores between individuals in the lowest vs highest total FACT-Cog score quartiles (Table S1).

\subsection{4 $\quad$ Covariates}

Potential confounding variables were age (continuous), race/ ethnicity (white non-Hispanic, and non-white), marital status (married or living as married; not married), word reading score as a marker of cognitive reserve (continuous score on the Wide Range Achievement Test Word Reading sub-test, fourth edition, or WRAT-4), receipt of radiation (yes; no), receipt of chemotherapy (yes; no), number of comorbidities, number of limitations to instrumental activities of daily living (IADLs), and study recruitment site. Because the study participants predominantly identified as white non-Hispanic, we could not further stratify the "non-white" group in statistical analyses.

\subsection{Statistical analysis}

Baseline descriptive characteristics of the study sample at baseline were calculated. We examined the unadjusted correlations between baseline APE, LM, and FACT-Cog scores (continuous) and baseline well-being domain scores (continuous). With separate models for each of the three cognitive measures, we used linear mixed-effects models to test the hypotheses that older breast cancer survivors with lower baseline APE, LM, and FACT-Cog scores would have persistently poorer global, functional, physical, social, and emotional domain scores over the 24-month follow-up. The linear mixed-effects models allowed us to account for the within-person correlations in the outcomes due to the repeated measures over time by incorporating subject-specific random effects. The models were adjusted for age, race/ethnicity, marital status, word reading score, receipt of radiation (yes vs no), receipt of chemotherapy (yes vs no), study recruitment site, in addition to a variable for time (in years) and a statistical interaction between time and the baseline cognitive measure, to allow the well-being outcome slopes (rate of change over time) to vary by baseline level of cognitive function.

Since study drop-out or death after the baseline can be informative with respect to the outcome, we calculated inverse probability of censoring weights (IPCWs) to account for any differential loss to follow-up due to study attrition or mortality. ${ }^{31}$ The IPCW is the inverse of the probability of study attrition through a given study time point, for person $i$ at time point $t$. We estimated these probabilities using logistic regression models including baseline covariates as predictors of study attrition: age, race/ethnicity, marital status, study recruitment site, pre-diagnosis self-reported physical function, fatigue symptom score, number of comorbidities, number of prescription medications, FACT-Cog score, depressive symptom score, and timed get-up-and-go. All models incorporated the IPCWs.

TAB LE 1 Baseline characteristics, Thinking and Living with Cancer Study, 2010 to 2016, N = 397

\begin{tabular}{|c|c|}
\hline Baseline characteristic & $\begin{array}{l}\text { Total N (\%) or } \\
\text { Mean (SD) }\end{array}$ \\
\hline Age (Mean; SD) & $68.0(6.0)$ \\
\hline Years of education (Mean; SD) & $15.2(2.2)$ \\
\hline \multicolumn{2}{|l|}{ Race/ethnicity } \\
\hline White (Non-Hispanic) & $314(79.1 \%)$ \\
\hline Non-White & $83(20.9 \%)$ \\
\hline \multicolumn{2}{|l|}{ Marital status } \\
\hline Married or living as married & $229(60.6 \%)$ \\
\hline Not married & $149(39.4 \%)$ \\
\hline WRAT-4 literacy score (Mean; SD) & $111.5(15.3)$ \\
\hline Number of comorbidities (Mean; SD) & $2.6(1.9)$ \\
\hline Number of IADL limitations (Mean; SD) & $0.44(1.02)$ \\
\hline \multicolumn{2}{|l|}{ Fatigue scale score } \\
\hline Mean (SD) & $43.15(8.49)$ \\
\hline \multicolumn{2}{|l|}{ Presence of depressive symptoms } \\
\hline No & $306(86.9)$ \\
\hline Yes & $46(13.1)$ \\
\hline \multicolumn{2}{|l|}{ Anxiety scale score } \\
\hline Mean (SD) & $29.2(8.2)$ \\
\hline \multicolumn{2}{|l|}{ Treatment modality } \\
\hline Chemo +/- HT & $111(29.0)$ \\
\hline HT only & $272(71.0)$ \\
\hline \multicolumn{2}{|l|}{ AJCC tumor stage } \\
\hline Stage 0 & $50(12.7)$ \\
\hline Stage 1 & $214(54.3)$ \\
\hline Stage 2 & $109(27.7)$ \\
\hline Stage 3 & $21(5.3)$ \\
\hline $\begin{array}{l}\text { FACT-G Global well-being score (Mean; } \\
\text { SD) }\end{array}$ & $81.15(12.86)$ \\
\hline $\begin{array}{l}\text { FACT-G Functional well-being score } \\
\text { (Mean; SD) }\end{array}$ & $74.43(20.22)$ \\
\hline $\begin{array}{l}\text { FACT-G Physical well-being score (Mean; } \\
\text { SD) }\end{array}$ & $83.37(16.27)$ \\
\hline $\begin{array}{l}\text { FACT-G Social well-being baseline score } \\
\text { (Mean; SD) }\end{array}$ & $85.96(16.88)$ \\
\hline $\begin{array}{l}\text { FACT-G Emotional well-being baseline } \\
\text { score (Mean; SD) }\end{array}$ & $82.04(17.23)$ \\
\hline
\end{tabular}

Note: US general adult female population FACT-G norms, scaled from 0 to 100, are: 73.7 (global), 65.35 (functional), 78.93 (physical), 70.71 (social), and 80.83 (emotional). US adult female cancer survivor FACT-G norms, scaled from 0 to 100, are: 76.02 (global), 69.64 (functional), 77.14 (physical), 79.64 (social), and 77.92 (emotional). From Brucker et al, 2005. ${ }^{18}$ 
For each model set, we extracted the predicted adjusted mean values and their $95 \%$ confidence intervals for each well-being outcome scale at each time point, and estimated the differential between the highest and lowest baseline cognitive function quartiles for each time point. We graphically presented change over time for each of the well-being outcomes according to baseline cognitive function quartiles and added the corresponding fully adjusted slope for the healthy cancer-free controls, to visually benchmark the degree of change over time in well-being outcomes that may be attributable to breast cancer, over and above aging over time alone. All statistical analyses were completed using SAS 9.0 (SAS Institute, Cary, NC).

\subsection{1 | Sensitivity analyses}

We conducted sensitivity analyses with mixed-effects models that were adjusted for our original model covariates, plus two iterative adjustments for baseline physical health and mental health indicators: first, we included number of comorbidities and limitations to instrumental activities of daily living (IADLs), and second, we included depressive symptom score (Center for Epidemiologic Studies Depression Scale) and anxiety symptom score (State-Trait Anxiety Inventory Scale). Because the causal directions of associations between these factors and cognitive function remain uncertain and could be bidirectional, ${ }^{20,21}$ we consider these analyses exploratory and hypothesis-generating for future studies.

\section{3 | RESULTS}

The mean age of the breast cancer survivors was 68 years (SD: 6 years; range: $60-98$ years). Mean years of education was 15.2 years (SD: 2.2 years), and $79.1 \%$ reported that they were white and nonHispanic (Table 1). Table 2 shows the unadjusted correlation

TABLE 2 Unadjusted correlation coefficients between baseline APE, LM, and FACT-Cog scores with global and domain-specific well-being scores, Thinking and Living with Cancer Study, 2010 to 2016, N = 397

\begin{tabular}{|c|c|c|c|c|c|}
\hline Baseline cognitive score & \multicolumn{5}{|c|}{ Baseline well-being score } \\
\hline APE & 0.11 & 0.13 & 0.14 & 0.02 & -0.01 \\
\hline$P$-value & (.03) & (.02) & (.01) & (.70) & (.92) \\
\hline LM & 0.08 & 0.12 & 0.10 & -0.01 & 0.06 \\
\hline$P$-value & (.11) & (.12) & (.05) & $(.82)$ & $(.27)$ \\
\hline$P$-value & $(<.0001)$ & $(<.0001)$ & $(<.0001)$ & $(.004)$ & $(<.0001)$ \\
\hline
\end{tabular}

Note: The well-being outcomes are scaled from 0 (low) to 100 (high).

Abbreviations: APE, attention processing speed, and executive function; LM, learning and memory.

(A)

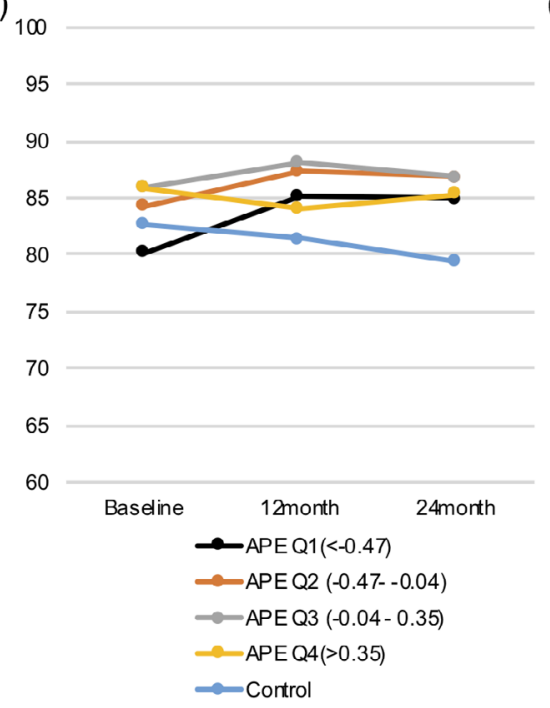

(B)

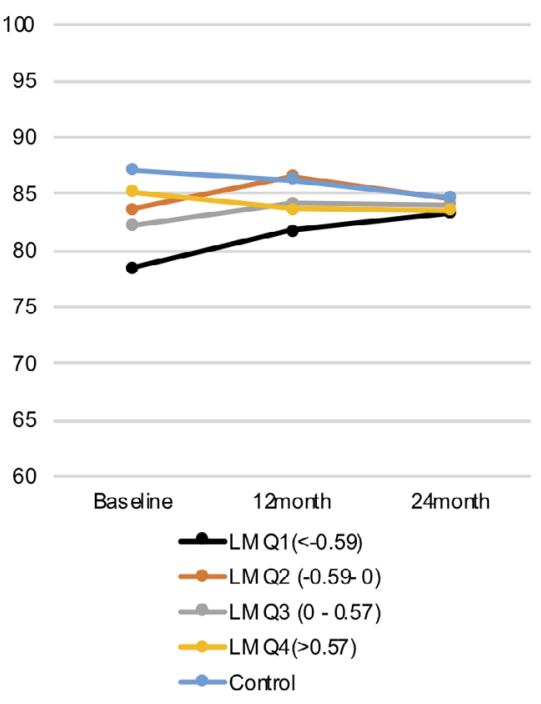

(C) ${ }_{100}$

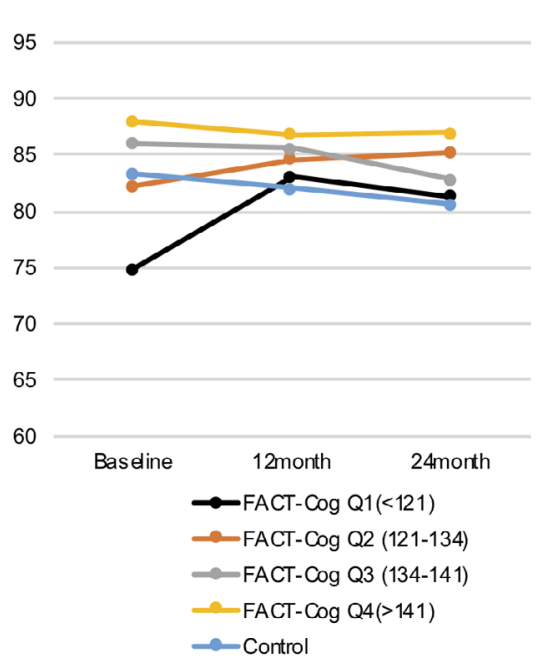

FIGURE 2 Adjusted global well-being scores over 24 months of follow-up, according to baseline attention, processing speed, and executive function quartile (Panel A), baseline learning and memory quartile (Panel B), and baseline FACT-Cog quartile (Panel C), all with cancer-free controls included for comparison 
TAB LE 3 Differences in mean 24-month well-being scores according to baseline cognitive measures, Thinking and Living with Cancer Study, 2010 to $2016, N=397$

\begin{tabular}{|lllllll}
\hline & \multicolumn{2}{l}{ Adjusted mean 24-month well-being score $(95 \% \mathrm{Cl})$} \\
\cline { 2 - 6 } Baseline cognitive measure & Global well-being & Functional well-being & Physical well-being & Social well-being & Emotional well-being \\
\hline APE (Q4-Q1 difference) & $-0.01(-5.59,5.58)$ & $-5.89(-14.48,2.69)$ & $-0.72(-6.6,5.15)$ & $8.67(-0.61,17.95)$ & $-0.93(-6.75,4.89)$ \\
\hline P-value & .997 & .178 & .809 & .067 & .754 \\
\hline LM (Q4-Q1 difference) & $0.41(-5.09,5.91)$ & $-1.32(-9.85,7.21)$ & $1.51(-4.25,7.27)$ & $0.96(-8.28,10.2)$ & $0.56(-5.02,6.15)$ \\
\hline P-value & .884 & .761 & .607 & .838 & .843 \\
\hline FACT-Cog (Q4-Q1 difference) & $6.31(1.49,11.14)$ & $5.97(-1.61,13.56)$ & $5.41(0.3,10.53)$ & $8.64(0.4,16.89)$ & $5.95(1.06,10.84)$ \\
\hline P-value & .011 & .122 & .038 & .040 & .017 \\
\hline
\end{tabular}

Note: All estimates are adjusted for baseline age, race/ethnicity, marital status, WRAT-4 literacy score, study recruitment site, receipt of radiation, receipt of chemotherapy, time (in years) and the interaction between time (in years) and the baseline cognitive measure. Inverse probability of censoring weights (IPCWs) were applied to all models to account for differential loss-to study follow-up. The well-being outcomes are scaled from 0 (low) to 100 (high). Abbreviations: APE, attention, processing speed, and executive function; LM, learning and memory; Q4, quartile 4 (highest); Q1, quartile 1 (lowest).

coefficients between baseline APE, LM, and FACT-Cog scores with baseline well-being domain scores.

\subsection{Cognition and global well-being}

Figure 2 shows the longitudinal trends in fully adjusted global wellbeing scores, according to baseline APE (Panel A), LM (Panel B), and FACT-Cog quartiles (Panel C). The model generating the estimates for this figure included the cancer-free controls to provide a visual benchmark for comparison with the trends in survivors. As shown in Figure 2, at baseline, the adjusted mean global well-being scores going from the highest to lowest APE quartile were: 85.9 (SE: 1.29), 85.9 (SE: 1.78), 84.3 (SE: 1.84), and 80.1 (SE: 1.83). For LM quartiles, the adjusted mean global well-being values were: 85.1 (SE: 1.29), 82.2 (SE: 1.68), 83.7 (SE: 1.82), and 78.5 (SE: 1.98). For FACT-Cog quartiles, the adjusted mean global well-being values were 87.99 (SE: 1.20), 86.03 (SE: 1.88), 82.26 (SE: 1.64), and 74.90 (SE: 1.78) (Figure 1). The differences in adjusted baseline global well-being between survivors in the lowest vs highest APE, LM, and FACT-Cog quartiles were 5.8, 6.6, and 13.1 points out of 100 , respectively, which were all clinically meaningful differences. At the 24-month follow-up, there were no statistically or clinically meaningful differences in global well-being according to baseline APE or LM scores (Table 3; Table S2). The mean predicted 24-month global well-being score in the highest baseline FACT-Cog quartile was 86.6 (95\% Cl: 83.1-90.1), and in the lowest FACT-Cog quartile was 80.3 (95\% Cl: 76.2-84.3), a clinically meaningful difference of 6.3 points ( $95 \% \mathrm{Cl}: 1.5$, 11.1; Table 3; Table S2).

\section{2 | Cognition and domain-specific well-being}

Changes over time in the functional and physical well-being domains were similar to those observed for global well-being for all three cognitive measures (Figures S1 and S2). The fully adjusted baseline differences between the highest vs lowest FACT-Cog quartiles were considered clinically meaningful for these two domains (16.8 for functional well-being; 16.4 for physical well-being; Figures S1 and S2). Social well-being declined over time and emotional well-being improved over time, on average, among the breast cancer survivors (Figures S3 and S4). At the 24-month follow-up, there were negligible differences in predicted well-being domain scores according to baseline APE and LM (Table 3). The predicted differences between the highest vs lowest baseline FACT-Cog score quartiles at the 24-month follow-up were statistically significant but not clinically meaningful for physical, social, and emotional well-being (Table 3).

\subsection{Sensitivity analyses}

When physical health indicators and mental health indicators were added to models, the physical health indicators generally changed the effect estimates very little, and the mental health indicators attenuated the effect estimates towards the null for the FACT-Cog (Table S3).

\section{4 | DISCUSSION}

This is the first large, prospective study of self-reported and objective cognitive function prior to systemic therapy and their relationships with changes in multi-domain well-being among older women over the first 2 years of breast cancer survivorship. Prior to systemic therapy, older breast cancer survivors with poorer objective and selfreported cognitive function had clinically meaningfully poorer global well-being scores, after accounting for key confounders. The women in this study tended to improve in well-being over time and the observed differences in well-being narrowed. However, there were persistent clinically meaningful differences in global well-being over time for women who reported the most cognitive impairments and lowest perceived cognitive abilities prior to their systemic therapy. Well-being was more closely related to self-reported cognitive 
function than to the neuropsychological test measures, consistent with prior research indicating that self-reported and objective cognitive function do not consistently correlate. ${ }^{24}$ We add new evidence indicating that, among older breast cancer survivors, self-reported cognitive function prior to systemic therapy is associated with a clinically meaningful decrement in global well-being over the first 24 months of survivorship.

\subsection{Comparison to other literature}

Our results are consistent with a cross-sectional study that correlated self-reports of cognitive function with well-being measures in older breast cancer survivors. ${ }^{32}$ We identified three studies that investigated cross-sectional and longitudinal associations between neuropsychological test scores and well-being among breast cancer survivors; ${ }^{17,18,33}$ however, their findings are inconsistent. Previous research has indicated that self-reported cognitive function is not only associated with depressive symptoms but also has relationships with well-being outcomes independently of depressive symptoms. ${ }^{29}$ In our sensitivity analysis, we observed that mental health indicators attenuated the relationship between self-reported cognitive function and well-being, but since these measurements were all taken at the same time, we cannot discern whether mental health is a confounder or mediator of this relationship. The role of mental health in the relationships between self-reported cognitive function and survivorship outcomes among older breast cancer survivors warrants further investigation.

\section{2 | Study limitations}

A limitation of this study is that it was conducted among mostly white, highly educated women who presented to mainly academic medical centers in urban areas. We observed higher mean global well-being scores in our sample relative to the established norms for the FACT scales (mean: 81.2; SD: 12.9 in our sample, vs mean: 73.7; SD: 17.2 in the US general adult female population and mean: 76.02; SD: 15.09 in the US adult female cancer survivor population), which was mostly driven by higher than average scores in the functional and social domains. ${ }^{27}$ The higher mean well-being scores that we observed may reflect the sociodemographic composition of our study, and we caution the generalization of our findings outside of this population subgroup. A high priority for future research on cancer and aging is to include diverse older population samples to improve the generalizability of findings, and to characterize and understand any potential health disparities in well-being and other outcomes that are relevant to older cancer survivors.

\subsection{Clinical implications}

In this study population, older women with relatively low objective and self-reported cognitive function prior to systemic therapy tended to recover somewhat in well-being over time, which should be a reassuring finding. However, older women who reported the lowest pre-systemic therapy cognition had clinically meaningful decrements in global well-being over the first 2 years of breast cancer survivorship. In this study, perceived cognitive impairments and perceived cognitive abilities were the sub-domains of self-reported cognitive function that appeared to primarily drive the observed associations. A next step is to determine whether older women who present with cognitive complaints at the time of their diagnosis might need detection and intervention to improve well-being over the course of cancer survivorship care. Our findings are consistent with literature supporting the use of geriatric assessments in oncology practice, which include brief self-reports of cognitive function. ${ }^{34-36}$

\section{\begin{tabular}{l|l}
4.4 & Conclusions
\end{tabular}}

Prior to systemic therapy, older breast cancer survivors with poorer objective and self-reported cognitive function had clinically meaningfully poorer global well-being scores, after accounting for key confounders. These women tended to improve in their well-being over time throughout the first 2 years of cancer survivorship. However, there were persistent clinically meaningful differences in global well-being over time for women who reported the most cognitive problems prior to systemic therapy. Well-being domains were more strongly associated with self-reported than objective cognitive function measures, supporting the clinical utility of self-reports of cognitive function for understanding well-being over the early phase of survivorship for older breast cancer survivors. The present study is a novel early contribution in this area, at a time when older cancer survivors make up an increasing share of the general population in the United States and elsewhere.

\section{ACKNOWLEDGMENTS}

We would like to thank the participants in the TLC study for sharing their time and experiences; without their generosity this study would not have been possible. We are also indebted to Sherri Stahl, Naomi Greenwood, Margery London, and Sue Peach who serve as patient advocates from the Georgetown Breast Cancer Advocates for their insights and suggestions on study design and methods to recruit and retain participants. We thank the following TLC study staff who contributed by ascertaining, enrolling, and interviewing participants: Chie Akiba, Anait Arsenyan, Jessica Bailey, Grace Butler, Savannah Carpenter, Caitlin Carr, Megan Chamberlain, Kemeberly Charles, Amy Chen, Jennifer Choi, Elana Cook, Julia Fallon, Maria Farberov, Robin Fatovic, Julie Filo, Alyssa Hoekstra, Mallory Hussin, Vani Katheria, Brittany Kennedy, Ty Lee, Abe Levi, Trina McClendon, Kat McNeal, Meghan Mihalache, Kelsey Obremski, Olivia O'Brian, Renee Ornduff, Elsa Roberts, Melissa Rose, Rupal Ramani, Ian SerVaas, Minna Song, Rebecca Wellner, Jessica Whitley, Rebecca Young, and Laura Zavala. This research was supported by the National Cancer Institute $(\mathrm{NCl})$ at the U.S. National Institutes of Health (NIH) grants R01CA129769 and R35CA197289 to JM. This study was also supported by the $\mathrm{NCl}$ grant 
P30CA51008 to Georgetown-Lombardi Comprehensive Cancer Center, and the Biostatistics and Bioinformatics and Non-Therapeutic Shared Resources at Lombardi Comprehensive Cancer Center. This research was also supported by NIH grants P30CA082709, P30AG10133, R01AG19771, and R01LM01136 (A.S. and B.M.); T32CA117865 and F31CA220964 (D.T.); Pepper Center Grant P30AG028716 (H.J.C.); and an institutional American Cancer Society grant IRG-92-152 to Georgetown-Lombardi Comprehensive Cancer Center (PI: Atkins) and R03CA241841 (L.C.K.).

\section{CONFLICT OF INTEREST}

The authors declare no conflicts of interest.

\section{DISCLOSURE}

The views expressed in this article are those of the authors and do not necessarily reflect the official views of the National Cancer Institute.

\section{DATA AVAILABILITY STATEMENT}

In accordance with the NIH Data Sharing Policy, de-identified data collected as part of this study and supporting documentation will be made available to other researchers who contact the Principal Investigator directly and complete a material transfer agreement.

\section{ORCID}

Lindsay C. Kobayashi (D) https://orcid.org/0000-0003-2725-3107

Brent J. Small (D) https://orcid.org/0000-0002-7444-4689

Danielle Tometich (D) https://orcid.org/0000-0002-5939-0635

James C. Root (D) https://orcid.org/0000-0001-7332-6165

\section{REFERENCES}

1. Bluethmann S, Mariotto A, Rowland J. Anticipating the "silver tsunami": prevalence trajectories and comorbidity burden among older cancer survivors in the United States. Cancer Epidemiol Biomark Prev. 2016;25:1029-1036.

2. Miller K, Siegel R, Lin C, et al. Cancer treatment and survivorship statistics, 2016. CA Cancer J Clin. 2016;66:271-289.

3. American Cancer Society. Cancer Treatment \& Survivorship: Facts \& Figures 2016-2017. Atlanta; 2016.

4. Hodes RJ, Sierra F, Austad SN, et al. Disease drivers of aging. Ann N Y Acad Sci. 2016;1386(1):45-68.

5. Hoeijmakers JH. DNA damage, aging, and cancer. N Engl J Med. 2009; 361:1475-1485.

6. Kuh D. The new dynamics of ageing (NDA) preparatory network. A life course approach to healthy aging, frailty, and capability. J Gerontol A Biol Sci Med Sci. 2007;62(7):717-721.

7. D'Orsi E, Xavier AJ, Steptoe A, et al. Socioeconomic and lifestyle factors related to instrumental activity of daily living dynamics: results from the English longitudinal study of ageing. J Am Ger Soc. 2014;62 (9):1630-1639.

8. Andreotti C, Root JC, Ahles TA, McEwan BS, Compas BE. Cancer, coping, and cognition: a model for the role of stress reactivity in cancer-related cognitive decline. Psychooncology. 2015;24(6):617-623.

9. Mandelblatt JS, Stern RA, Luta G, et al. Cognitive impairment in older patients with breast cancer before systemic therapy: is there an interaction between cancer and comorbidity? J Clin Oncol. 2014;32(18): 1909-1918.
10. Kohli S, Griggs JJ, Roscoe JA, et al. Self-reported cognitive impairment in patients with cancer. J Oncol Pract. 2007;3(2):54-59.

11. Mandelblatt JS, Hurria A, McDonald BC, et al. Cognitive effects of cancer and its treatments at the intersection of aging: what do we know; what do we need to know? Semin Oncol. 2013;40(6):709-725.

12. Ahles TA, Hurria A. Cognition and cancer: conceptual and methodological issues and future directions. Psychooncology. 2018;27:3-9.

13. Loh KP, Janelsins MC, Mohile SG, et al. Chemotherapy-related cognitive impairment in older patients with cancer. J Geriatr Oncol. 2016;7 (4):270-280.

14. Sedrak MS, Hurria A. Cancer in the older adult: implications for therapy and future research. Cancer. 2018;124(6):1108-1110.

15. Hurria A, Soto-Perez-de-Celis E, Allred JB, et al. Functional decline and resilience in older women receiving adjuvant chemotherapy for breast cancer. J Am Ger Soc. 2019;67(5):920-927.

16. Shilling V, Jenkins V. Self-reported cognitive problems in women receiving adjuvant therapy for breast cancer. Eur J Oncol Nurs. 2007; 11(1):6-15.

17. Reid-Arndt S, Hsieh C, Perry M. Neuropsychological functioning and quality of life during the first year after completing chemotherapy for breast cancer. Psychooncology. 2010;19(5):535-544.

18. Mehnert A, Scherwath A, Schirmer L, et al. The association between neuropsychological impairment, self-perceived cognitive deficits, fatigue and health related quality of life in breast cancer survivors following standard adjuvant versus high-dose chemotherapy. Patient Educ Couns. 2007;66(1):108-118.

19. Jenkins V, Shilling V, Deutsch G, et al. A 3-year prospective study of the effects of adjuvant treatments on cognition in women with early stage breast cancer. Br J Cancer. 2006;94(6):828-834.

20. Donovan NJ, Wu Q, Rentz DM, Sperling RA, Marshall GA, Glymour MM. Loneliness, depression and cognitive function in older U.S. adults. Int J Geriatr Psychiatry. 2017;32(5):564-573.

21. Airaksinen E, Larsson M, Lundberg I, Forsell Y. Cognitive functions in depressive disorders: evidence from a population-based study. Psychol Med. 2004;34:83-91.

22. Pullens MJJ, De Vries J, Roukema JA. Post-treatment subjective cognitive dysfunction in breast cancer patients: a systematic review. Psychooncology. 2009;19:1127-1138.

23. Bray VJ, Dhillon HM, Vardy JL. Systematic review of self-reported cognitive function in cancer patients following chemotherapy treatment. J Cancer Surviv. 2018;12(4):537-559.

24. Ganz PA, Kwan L, Castellon SA, et al. Cognitive complaints after breast cancer treatments: examining the relationship with neuropsychological test performance. J Natl Cancer Inst. 2013;105(11):791-801.

25. Mandelblatt JS, Small BJ, Luta G, et al. Cancer-related cognitive outcomes among older breast cancer survivors in the thinking and living with cancer study. J Clin Oncol. 2018;36:3211-3222. https://doi.org/ 10.1200/JCO.18.00140 [Epub ahead of print].

26. Webster K, Cella D, Yost K. The functional assessment of chronic illness therapy (FACIT) measurement system: properties, applications, and interpretation. Health Qual Life Outcomes. 2003;1:79.

27. Brucker PS, Yost K, Cashy J, Webster K, Cella D. General population and cancer patient norms for the functional assessment of cancer therapy-general (FACT-G). Eval Health Prof. 2005;28(2):192-211.

28. Cella D, Tulsky D, Gray G, et al. The functional assessment of cancer therapy scale: development and validation of the general measure. J Clin Oncol. 1993;11(2):570-579.

29. Van Dyk K, Hunter AM, Ercoli L, Petersen L, Leuchter AF, Ganz PA. Evaluating cognitive complaints in breast cancer survivors with the FACT-cog and quantitative electroencephalography. Breast Cancer Res Treat. 2017;166(1):157-166.

30. Cheung YT, Foo YL, Shwe M, et al. Minimal clinically important difference (MCID) for the functional assessment of cancer therapy: cognitive function (FACT-cog) in breast cancer patients. J Clin Epidemiol. 2014;67(7):811-820. 
31. Cole SR, Hernán MA. Constructing inverse probability weights for marginal structural models. Am J Epidemiol. 2008;168(6):656-664.

32. Von Ah D, Tallman EF. Perceived cognitive function in breast cancer survivors: evaluating relationships with objective cognitive performance and other symptoms using the functional assessment of cancer therapy-cognitive function instrument. J Pain Symptom Manag. 2015;49(4):697-706.

33. Reid-Arndt S, Yee A, Perry M, Hsieh C. Cognitive and psychological factors associated with early post-treatment functional outcomes in breast cancer survivors. J Psychosoc Oncol. 2009;27(4):415-434.

34. Williams GR, Dunham L, Chang Y, et al. Geriatric assessment to predict hospitalization frequency and long-term care utilization in older adult cancer survivors. J Clin Oncol. 2019;15(5):e399-409.

35. Magnuson A, Allore $\mathrm{H}$, Cohen $\mathrm{HJ}$, et al. Geriatric assessment with management in cancer care: current evidence and potential mechanisms for future research. J Geriatr Oncol. 2016;7(4):242-248.

36. Soto-Perez-de-Celis E, Li D, Yuan Y, Lau YM, Hurria A. Functional versus chronological age: geriatric assessments to guide decision making in older patients with cancer. Lancet Oncol. 2018;19(6): e305-16.

\section{SUPPORTING INFORMATION}

Additional supporting information may be found online in the Supporting Information section at the end of this article.

How to cite this article: Kobayashi LC, Cohen HJ, Zhai W, et al. Cognitive function prior to systemic therapy and subsequent well-being in older breast cancer survivors: Longitudinal findings from the Thinking and Living with Cancer Study. Psycho-Oncology. 2020;29:1051-1059. https:// doi.org/10.1002/pon.5376 